\title{
Kinematics of the Filaments in the Crab Nebula
}

\author{
G. MÜNCH \\ Mount Wilson and Palomar Observatories, Carnegie Institution of Washington, California \\ Institute of Technology, Pasadena, California
}

$T$ HE Crab Nebula, reinnant of the supernova of 1054 , may be observed today in the recombination spectrum of the filamentary component, or in the continuous spectrum of the central amorphous mass. The recent optical discovery of a high degree of polarization in the amorphous component has led to a striking confirmation and general acceptance of the hypothesis first advanced by Shklovsky, ${ }^{1}$ which considers the continuous spectrum as emitted by relativistic electrons moving in a magnetic field.

I want to call attention to some properties of the motion of the filamentary mass which may have some bearing on the problems of the structure of the nebula as a whole and of the origin of its magnetic field. In order to bring into context the result of my observations, it will be necessary to outline briefly the status of the problem. Under the fundamental hypothesis that the continuous radiation of the nebula is produced by the acceleration of high-energy electrons, it is a fairly straightforward problem to evaluate the total number of electrons, with the appropriate energy, required to produce the observed power in the photographic and radio regions of the spectrum. Oort and Walraven ${ }^{2}$ have estimated in this fashion that the total amount of energy $E_{f e}$ contained by the fast electrons of the nebula is of the order of $10^{48}$ ergs. The decay time of the electrons emitting the visible light (energies around $10^{11}$ ev) is of the order of 200 years; thus, since the supernova explosion took place 900 years ago, they must be continuously being renovated. Using an argument which essentially invokes an equipartition between the energy density of the free electrons and the energy density of the magnetic field, Oort and Walraven ${ }^{2}$ estimated that the magnetic field strength $H$ required is near $10^{-3}$ gauss. Values of $H$ and $E_{f e}$ of the same order of magnitude have been derived by Pickelner. ${ }^{3}$ The amount of energy $E_{f_{e}}$ is surprisingly large, as it represents between 1 and $10 \%$ of the total energy radiated in the supernova explosion. It compares with the gravitational energy of a star of solar dimensions $\left(7 \times 10^{48} \mathrm{ergs}\right)$ and the kinetic energy of mass motion present in the filamentary component (mass $=2 \times 10^{32} \mathrm{~g}$, $\left.\left\langle V^{2}\right\rangle^{\frac{1}{2}}=10^{8} \mathrm{~cm} / \mathrm{sec}\right)$. And finally it is only $10^{-4}$ times the total nuclear energy available in the sun from the conversion of hydrogen into helium. It should be kept in mind, moreover, that the contribution by protons

${ }^{1}$ I. S. Shklovsky, Doklady Akad. Nauk S.S.S.R. 90, 983 (1953).

2 J. H. Oort and T. Walraven, Bull. Astron. Inst. Neth. 12, 285 (1956).

${ }_{3}^{3}$ S. B. Pickelner, Astron. Zhur. 33, 785 (1956). and heavier particles to the total energy of the fast particles is not known with any certainty. Oort and Walraven ${ }^{2}$ suppose that the energy of the protons equals that of the electrons. But as Burbidge ${ }^{4}$ has pointed out, in any kind of acceleration mechanism of the induction type, the relative energy of protons and electrons would be in the ratio of their masses if the electrons were of primary origin. It is well known that electrons are more difficult to accelerate because of their large energy losses in the low-energy range by bremsstrahlung and ionization processes. In a detailed study of the acceleration process, therefore, it is necessary to consider mechanisms of electron and positron production, following nuclear collisions between heavy particles, as Burbidge ${ }^{4}$ has attempted to do. The various meson decay constants involved in the reactions are rather uncertain, but in any event it seems likely that the energy of the electrons is less than that of the protons. The discussion of this particular question belongs to the field of nuclear physics rather than to gas dynamics, but it is important to realize that the energy contained by all the fast particles may be considerably in excess of the value suggested by Oort and Walraven. ${ }^{2}$

Among the many problems related to the Crab Nebula the origin of the magnetic field is of the greatest importance in hydromagnetic studies. Shklovsky ${ }^{1}$ and Ginzburg ${ }^{5}$ suggested that the magnetic field might be of interstellar origin. Oort and Walraven ${ }^{2}$ did not consider this explanation likely and instead advanced the hypothesis that it might be generated by some sort of dynamo effect of the motion of the filaments. Still a third possibility is that the field may be a relic of that present in the parent star of the supernova in the pre-explosion stage. As Chandrasekhar suggested in the Guanajuato Cosmic Ray Conference, if the present field were compressed into a star of solar dimensions, the resulting field would be so large as to make the star unstable magnetically. This last idea would provide not only an explanation for the origin of the magnetic field but also a mechanism for the supernova explosion itself. (Editorial note: The problem of the origin of the field seems to have been deferred.)

In relation to the origin of the magnetic field, it is appropriate to point out an obvious structural feature of the Crab Nebula, which as far as I have been able to find out, has not been noticed before. Namely, the

${ }^{4}$ G. R. Burbidge, Astrophys. J. 124, 416 (1956).

5 V. L. Ginzburg, Uspekhi Fiz. Nauk 51, 343 (1953). 


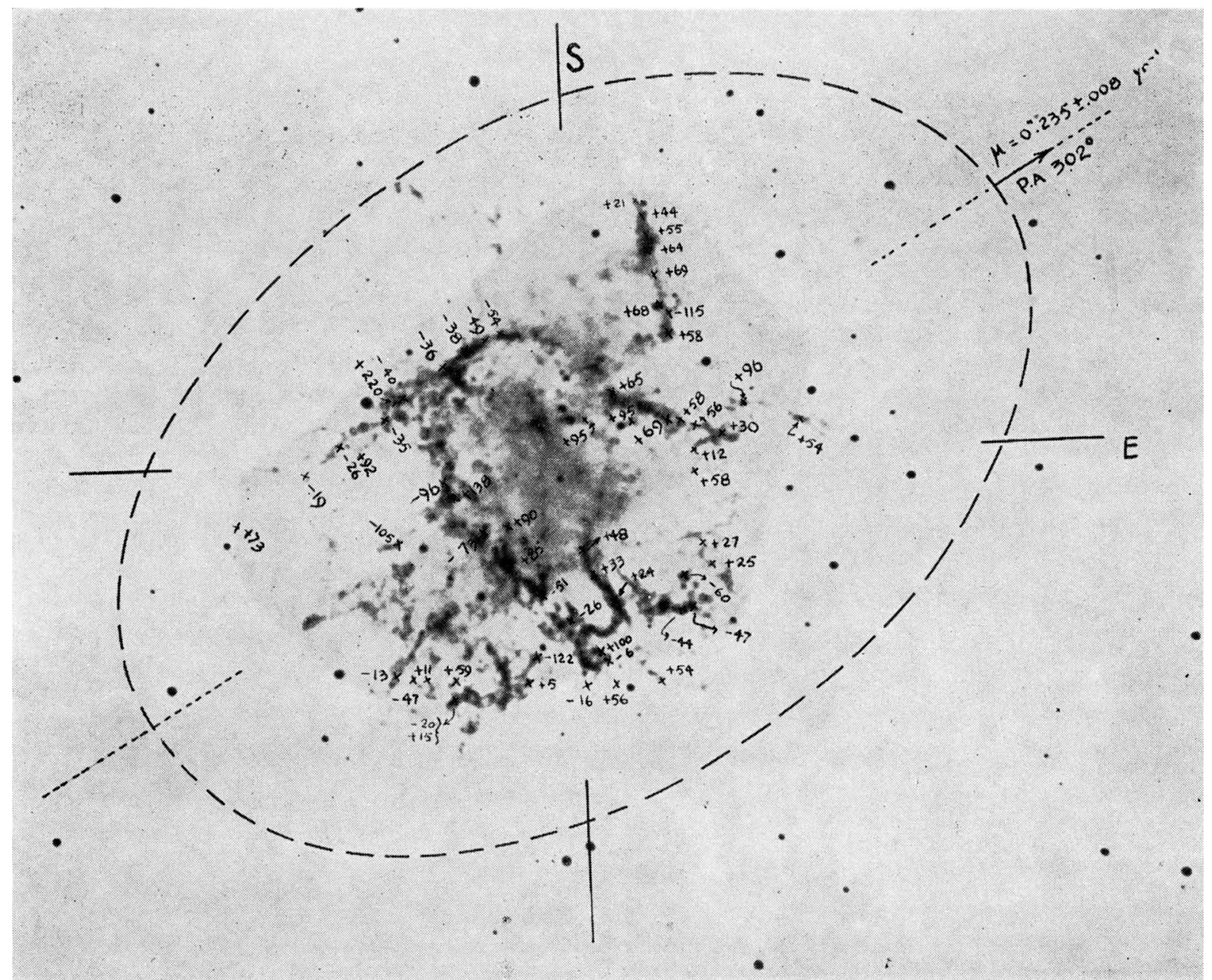

FIG. 1. Radial velocities of the brighter filaments in the Crab Nebula, expressed in units of $10 \mathrm{~km} / \mathrm{sec}$. The envelope of the outer filaments has been drawn from a long exposure red plate obtained by W. Baade.

direction of the major axis of the nearly elliptical envelope of the filaments is that of the galactic equator at that point in the sky (Fig. 1). The coincidence is as close as can be expected from the uncertainty with which a major axis can be determined for the envelope curve (between $1^{\circ}$ and $2^{\circ}$ ). It would seem hardly a matter of chance, and suggests immediately either a very unlikely symmetry in the ejection process which gave origin to the supernova or an effect of the surrounding interstellar material. The latter possibility results from considering the effects of the magnetic field prevalent in the general interstellar medium. In the theory of Davis and Greenstein ${ }^{6}$ for the alignment of the interstellar dust grains, a mean field of $10^{-5}$ gauss in the galactic plane is required to explain the observed interstellar polarization of distant stars. The observations by Hiltner $^{7}$ of stars near the Crab Nebula indicate that the lines of force of the interstellar field in this general direction are very nearly in the galactic

${ }^{6}$ L. Davis and J. L. Greenstein, Astrophys. J. 114, 206 (1951) ${ }^{7}$ W. A. Hiltner, Astrophys. J., Suppl. Ser. 1, 389 (1956). plane. Since the linear scale of the irregularities present in the interstellar magnetic field are much larger than the dimensions of the nebula, we would expect that an initially symmetric explosion would advance against the surrounding medium with a velocity depending on the direction of the external magnetic field. It would be a maximum in the directions of the lines of force, which we have found to coincide with the maximum extension of the nebula, and a minimum in the perpendicular direction. The external magnetic field will have an appreciable effect on the material motion only when its pressure $H_{e}{ }^{2} / 8 \pi$ reaches a value comparable with the kinetic energy density of the shell $(v>1000 \mathrm{~km} / \mathrm{sec})$, or $H_{e} \approx 10^{-3}$ gauss. Whether the field strength may reach so high a value, from an initial value one hundred times less, depends on how fast the internal energy in the compressed zone may be radiated away. In any case, it is clear that if the external field had been built up to the extent of holding the expansion down in the direction of the minor axis, then the present velocity of expansion at right angles to the major axis would be less 


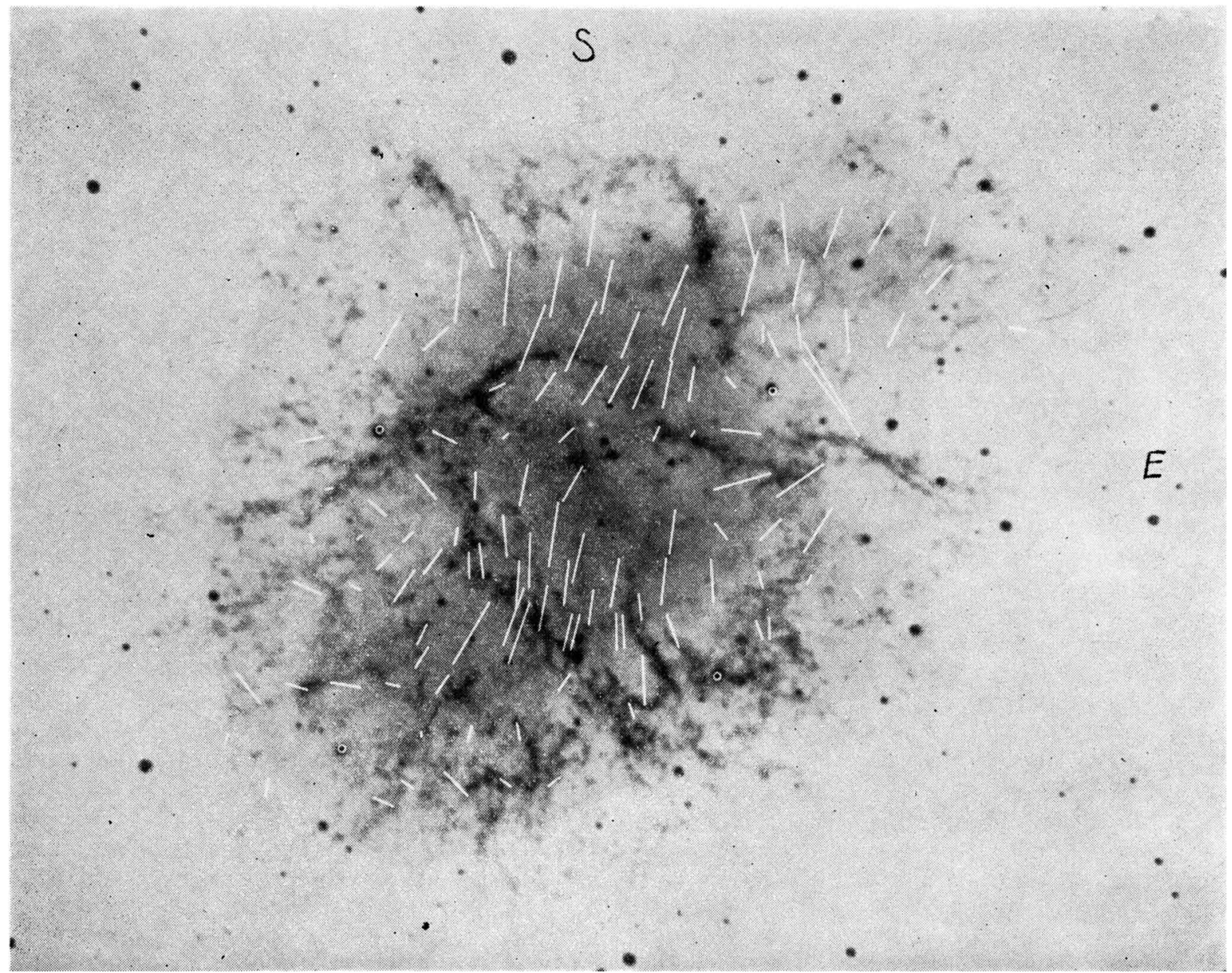

FIG. 2. Composite photograph illustrating the relation between the direction of the filaments and that of the plane of vibration of the electric vector of the synchrotron radiation, as measured by W. A. Hiltner.

than its initial value. Consequently, the distance of the nebula, derived from the equality of the angular motion in the plane of the sky (mostly along the major axis) with the radial component of the velocity, may be an underestimate. It will be possible to find the deceleration of the shell when proper motions and radial velocities of filaments near the extreme of the minor axis are determined in a more accurate fashion than has been done in the past.

As mentioned already, the kinetic energy of the expanding mass of filaments is of the same order of magnitude as the energy contained in the magnetic field. One would expect then that the magnetic field will affect strongly the motions of the filaments. Conversely, detailed knowledge of the motion of the filaments will provide some information about the magnetic field. With this idea in mind, I started determining radial velocities of individual filaments in the nebula by the Doppler shifts of the lines $\lambda \lambda 6716$ and 6583 of [NII], $\mathrm{H} \alpha$, and $\lambda \lambda 6716$ and 6730 of [SII]. The observations have been made with the prime focus spectrograph of the 200-inch telescope with a solid
Schmidt camera of 3 inches focal length and a 7500 lines/inch grating in the third-order spectrum. The linear dispersion of the plates, $110 \mathrm{~A} / \mathrm{mm}$, and the angular resolving power in the plate, $36^{\prime \prime} .6 / \mathrm{mm}$, are considerably larger than that employed in previous observations. Sor far only the brighter central filaments have been observed. The number of condensations is so large that even at this large dispersion it is not always possible to identify unambiguously in the direct photograph the filament to which an observed set of spectral lines corresponds. A sample of the measured velocities is given in Fig. 1 , where the radial velocities, in units of $10 \mathrm{~km} / \mathrm{sec}$, have been drawn at corresponding points in the direct photograph. A large range in velocities is present, from a maximum of +2200 $\mathrm{km} / \mathrm{sec}$ to a minimum of $-1150 \mathrm{~km} / \mathrm{sec}$. On the whole, however, it may be noticed that the more elongated filaments show some kinematic continuity also. In particular the ring which seems to be nearly contained in a plane normal to the major axis stands as a unit, with its SE extreme receding and its NW arc approaching the observer. Along a given filament there are 
considerable fluctuations in the radial velocity. For example, along the filament running almost straight $\mathrm{NS}$, in the south following quadrant, we observe a range in velocity from +440 to $+690 \mathrm{~km} / \mathrm{sec}$ (disregarding a point at $-1150 \mathrm{~km} / \mathrm{sec}$, which obviously corresponds to another unit). These velocity differences should be compared with the internal velocity dispersion, which may be estimated from the line widths, observed at those places where we can be reasonably certain that there is no superposition along the line of sight. Spectra obtained with the 200 -inch Coudé spectrograph and the $f: 0.67$ aplanatic camera show the lines as sharp as the instrumental contour, which has a spread equal to the projected slit width and at $\mathrm{H} \alpha$ amounts to $52 \mathrm{~km} / \mathrm{sec}$. The internal velocity dispersion presumably is less than one half this amount.

The orientation of the filaments in the plane of the sky shows an immediate relation to the direction of polarization of the continuous radiation. In Fig. 2 we have drawn on the $\mathrm{H} \alpha$ direct photograph the direction of the plane of vibration of the electric vector of the continuous radiation, as measured by Hiltner ${ }^{8}$ through a $15^{\prime \prime}$ diaphragm. It is noticed that many of the filaments are oriented in a direction nearly parallel to

${ }^{8}$ W. A. Hiltner, Astrophys. J. 125, 300 (1957). that of the electric vector. Since we can observe only the continuous radiation of the electrons moving directly towards the observer, the direction of the magnetic lines of force contained in the plane of the sky are then normal to the directions of the filaments also approximately contained in the same plane. It is then suggestive to think that the filaments move in a force-free field, in which the current density $\mathbf{j}$ is parallel to the magnetic field strength, making the Lorentz force, curl $\mathbf{H} \times \mathbf{H}$, vanish. The presence of the magnetic field would not be then affecting the motions, indicating that a nearly stationary state has been reached.

The observational evidence we have presented here would indicate a close connection between the magnetic field and the motion of the filaments. The suggestion of Oort and Walraven ${ }^{2}$ regarding the origin of the magnetic field from a dynamo effect of the motion of the filaments receives thus some support. On the other hand, the effect that the galactic field may have on the structure of the outer shell of filaments brings into the picture also the relevance of the earlier hypothesis of Shklovsky ${ }^{1}$ and Ginzberg. ${ }^{5}$ Further study of the problem may show that the magnetic field observed at present in the Crab Nebula resulted through the operation of both mechanisms.

\section{DISCUSSION}

\begin{abstract}
A. SCHLÜTER, Max Planck Institut für Physik, Göttingen, Germany: Given that the filaments are perpendicular to the magnetic field, there is a striking resemblance between these filaments and some aspects of solar filaments. According to a theory I have proposed for the solar filaments which has, I think, been substantiated by Babcock's measurements on his solar magnetograms, the filament is simply resting on a kink of the lines of force; that is to say, electric current is flowing through the filament parallel to the surface of the sun at exactly those places where the magnetic field without the presence of the filament would be horizontal (parallel to the surface of the sun). If we assume essentially the same mechanism to hold at the surface of the expanding region of the Crab Nebula, we have simply to replace gravitation in the solar case by acceleration in the Crab Nebula case. This matter in the Crab Nebula is under acceleration due to the pressure exerted by the expanding diffuse structureless inner part of the Crab Nebula, and the only stable position where it can be supported by the magnetic field is where the magnetic field of the inner part is somehow parallel to the surface of the expanding region. So, theoretically, the filament should form some kind of a belt or girdle around the equatorial belt of the expanding region.
\end{abstract}

S. B. PICKelneR, Crimean Astrophysical Observatory, Simeis, U.S.S.R.: Baade, 15 years ago, found from Duncan's measurements some acceleration, about $0.0011 \mathrm{~cm} / \mathrm{sec}^{2}$. This acceleration was found along the large axis of this nebula. If real, the acceleration may be explained as a consequence of the magnetic and cosmic-ray pressures because there are no other forces which can accelerate the expanding shell of a nova. The magnetic field may be about $3 \times 10^{-4}$ gauss, and the cosmic-ray pressure is to a first approximation the same as the magnetic pressure; from this we can compute the acceleration of this shell. From these two data may be computed the mass of the shell; this mass turns out to be about 0.1 solar mass. The elliptical form of the Crab Nebula may also be discussed. The internal magnetic field in the central region is directed along the minor axis and the tension of the magnetic lines of force may decrease the velocity expansion in this direction. The question about the relation between the filaments and magnetic field was discussed by Shklovsky in a recent paper.

The magnetic field is more uniform in the central part of the Crab Nebula, and the magnetic lines run through the shell and return to the other side of the 
uniform field. If the shell expands, the extension of the magnetic lines may give a deceleration of this part of the shell.

A. SCHLÜTER : I wish to point out two observational facts. In the case of the Barnard ring, which is almost elliptical in shape, the major axis is almost parallel to the galactic equator. In addition to that, there is another nebulosity around Sigma Orionis which is also elliptical and which also has the major axis almost parallel to the galactic equator. I do not know whether this is significant or not. 